\title{
Preparación Hospitalaria en incidentes NBQ
}

\author{
Cique Moya A. ${ }^{1}$
}

Sanid. mil. 2015; 71 (1): 44-49; ISSN: 1887-8571

\begin{abstract}
RESUMEN
Los incidentes de Goainia, Matsumoto, Tokio, así como la crisis del carbunco muestran que los hospitales intervienen de forma directa en la respuesta a incidentes NBQ, debiendo estar integrados en el sistema de respuesta a desastres junto con los servicios de asistencia prehospitalaria para poder dar una respuesta efectiva a este tipo de accidentes. Los retos organizativos y de gestión a los que tiene que hacer frente la estructura hospitalaria ante un incidente no sólo afectan a los servicios de urgencias, sino que trascienden a ésta, debiendo estar contemplados en el Plan de Emergencia del Centro Hospitalario. La respuesta pasa por el establecimiento de un plan de mejora continua en la instalación mediante la valoración del índice de seguridad de la instalación y la preparación, individual y colectiva, del centro sanitario para activar el plan de emergencias de forma inmediata una vez establecida la alarma, para así evitar los problemas de transferencia de contaminación a las instalaciones y al personal minimizando las consecuencias de un incidente NBQ.
\end{abstract}

PALABRAS CLAVE: Incidentes NBQ, Preparación hospitalaria, transferencia de la contaminación, plan de emergencias, índice de seguridad, análisis vulnerabilidad.

\section{Hospital preparation in NBC incidents}

SUMMARY: Goiania, Matsumoto and Tokyo incidents, as well as the anthrax crisis show that the hospitals are directly involved in the response to NBC incidents, which is why they must be integrated into the system of response to disasters together with prehospital care services in order to provide an effective response to such accidents. Organizational and management challenges that hospital faces in an incident do not only affect emergency services, they go beyond, so they must be considered in the Hospital's Emergency Plan. The answer is the establishment of a plan for continuous improvement in the installation through the assessment of its safety index and through the preparation, individual and collective, of the health center in order to activate the emergency plan immediately once set the alarm, avoiding that way the transfer of contamination to facilities and staff and minimizing the consequences of a NBC incident.

KEYWORDS: NBC incident, Hospital preparation, Transfer of contamination, Emergency plan, Safety index, Vulnerability analysis

\section{INTRODUCCIÓN}

Goiania, Matsumoto, Tokio o el Amerithrax son claros ejemplos de cómo un centro sanitario puede verse afectado por un incidente no convencional como es un incidente NBQ, tanto de origen accidental como de origen provocado. Aunque hay que tener en cuenta que no tiene porqué producirse un incidente con múltiples víctimas para que un centro sanitario pueda ver afectado en un incidente no convencional, ya sea por un incidente individual o en un incidente con múltiples víctimas.

Goiania demostró la necesidad de preparación de los centros hospitalarios, en función de su nivel asistencial, para atender a los afectados por Síndrome de Radiación Aguda, tanto desde el punto de vista estructural y funcional, como de la necesidad de formación y entrenamiento específico del personal sanitario para reconocer y manejar a este tipo de pacientes ${ }^{1}$. Primando lo segundo sobre lo primero, ya que el rápido reconocimiento de

${ }^{1}$ Tcol. Veterinario. Instituto de Medicina Preventiva de la Defensa.

Dirección para correspondencia: Alberto Cique Moya. Servicio de Sanidad Ambiental y NBQ. Instituto de Medicina Preventiva de la Defensa. Glorieta del Ejército 1. 28047 Madrid. España

Recibido: 23de enero de 2014

Aceptado: 8 de octubre de 2014 los síntomas iniciales puede redundar en un mejor pronóstico, no sólo para los pacientes sino para su entorno al reducir el peligro de transferencia de la contaminación (exposición secundaria). Pudiendo entonces derivar hacia centros especializados los afectados en un incidente radiológico dentro de un criterio de economía de medios, de reconocimiento legal o de especialización profesional2-4.

El «incidente de Matsumoto» y el atentado de Tokio demostró que las víctimas en un incidente no convencional debían ser descontaminadas para evitar la transferencia de contaminación al conjunto de la cadena de rescate, ya que esta podía afectar no sólo a las propias víctimas, sino al personal de intervención e incluso al personal de los servicios de urgencias de los centros sanitarios donde eran derivadas ${ }^{5}$. Obligando a formarse y entrenarse, así como a adoptar el adecuado nivel de protección individual para poder intervenir ante un posible incidente de múltiples víctimas por agentes NBQ en cualquiera de los niveles asistenciales ${ }^{6,7}$. Motivo por el cual, se hace necesario realizar un esfuerzo de preparación frente a esta amenaza, ya tenga un origen accidental como tenga un origen intencionado ${ }^{8}$.

El Amerithrax (crisis del carbunco) manifestó la falta de preparación de los centros sanitarios frente al empleo intencionado de agente biológicos, detectándose carencias en la gestión de incidentes, desde la falta de comunicación entre los gestores y el 


\section{Preparación Hospitalaria en incidentes NBQ}

sistema de salud, hasta la necesidad de coordinación e integración de los diferentes servicios y especialidades, pasando por la necesidad de disponer de reservas de productos farmacéuticos. Sin olvidar la necesidad perentoria de formación del personal, en sus diferentes niveles, desde el personal de laboratorio hasta el de seguridad, desde el personal facultativo hasta el personal auxiliar para poder responder con eficacia a una emergencia sanitaria infecciosa de origen provocado?.

$\mathrm{Y}$ es que no se puede olvidar que los preparativos individuales y colectivos que se establezcan no sólo servirán para los desastres provocados por el hombre, ya sean accidentales o provocados, criminales o no, sino que también servirán y sirven de forma directa para enfrentarnos a la amenaza de las enfermedades emergentes o reemergentes, ya se trate del caso Litvinenko, un intento de autolisis, el Síndrome Agudo Respiratorio Grave o la gripe A donde el nivel asistencial requerido supone un reto para la organización sanitaria ${ }^{10-15}$.

\section{ANÁLISIS DE RIESGOS}

Los centros sanitarios están sujetos en mayor o menor medida a riesgos externos e internos, ya sean de origen natural, tecnológico o antrópico, incluyéndose en estos últimos al terrorismo NBQ, donde se producirán escenarios de baja mortalidad, pero con elevada morbilidad, con lo que se hace necesario un esfuerzo de preparación de los centros sanitarios en función de un riguroso análisis y caracterización de riesgos ${ }^{16}$. Para lo cual se debe intentar responder a una serie de interrogantes que deben ser respondidas en función del nivel de amenaza: ¿Hay amenaza NBQ? o si ¿Somos objetivo?.

Ambas cuestiones están íntimamente relacionadas, ya que a pesar de que el índice de vulnerabilidad frente al terrorismo NBQ en nuestro entorno pueda resultar bajo, no se puede olvidar la necesidad de prepararse frente a lo improbable. De hecho, la organización religiosa «Verdad Suprema» reveló a la sociedad que las Armas de Destrucción Masiva (ADM) no sólo estaban al alcance de los estados, sino que había organizaciones o individuos que eran capaces de alcanzar la capacidad operacional de diseminación de agentes NBQ y que estaban dispuestos a utili$\operatorname{zarlas}^{17,18}$.

Estas dos primeras preguntas llevan implícitas tres cuestiones derivadas ¿Se puede? ¿Se sabe? y/o ¿Se quiere? Debiendo ser conscientes que la respuesta sin preparación es el prologo al desastre, de ahí deban integrarse en el sistema de respuesta a incidentes NBQ todos los niveles asistenciales, desde la asistencia prehospitalaria a la hospitalaria; integrándose en el sistema de respuesta a emergencias NBQ los protocolos y procedimientos de intervención dentro del Plan de Emergencia o de Catástrofes del centro sanitario en función de la entidad del mismo o del sistema organizativo implantado en el área ${ }^{19}$.

Dentro de la categorización de emergencias el sistema sanitario debiera ser capaz de responder, con mayor o menor eficacia, a los desastres o catástrofes, sea cual sea el origen que la genera. Debiéndose adecuar los protocolos en función de la alarma y del número de víctimas generados en el incidente, primando la respuesta coordinada y progresiva en función de una economía de medios y de acuerdo al principio de precaución ${ }^{20}$. Aunque desde el punto de vista NBQ hay que tener en cuenta que una sola víctima puede provocar que el centro sanitario resulte afectado en su operatividad. Sirva de ejemplo la transferencia de contaminación de un paciente ingresado en urgencias tras un intento de autolisis con un compuesto organofosforado que provocó la transferencia de la contaminación a tres miembros del personal de urgencias que le estaban atendiendo, dejándoles inoperativos, hecho que probablemente no se hubiera producido si hubieran seguido las instrucciones del centro de toxicología que les había asesorado en lo relativo a descontaminación del paciente ${ }^{21}$.

Este incidente, y salvando las distancias con un incidente NBQ, nos muestra que la respuesta debe ser rápida, coordinada, proporcionada e integral. Los centros sanitarios deben ser capaces de activar de forma prácticamente inmediata, cuando todavía se está generando la alarma de un incidente NBQ, el plan de emergencias, para así ser capaces de dar una respuesta eficaz al incidente en función de la gravedad del mismo.

La triste experiencia alcanzada en atentados terroristas muestra que el tiempo inicial de llegada de víctimas en masa, la mayor de las veces por sus propios medios, tras un ataque terrorista suele situarse, aproximadamente, entre los 20 y 60 minutos de producido éste ${ }^{22,23}$. De hecho en el ataque iraquí sobre la ciudad de Hosseiniyeh con neurotóxicos, 300 afectados llegaron en los cinco minutos tras el ataque, alcanzando los 1700 en las siguientes horas; mientras que en el atentado de Tokio, el Hospital de St. Luke atendió a 641 personas el primer día y 349 en los siete siguientes, habiendo sido realizados el $93 \%$ de los traslados hacia los centros sanitarios por personal no sanitario en vehículos particulares ${ }^{24}$. De ahí la importancia de la inmediata activación del plan de emergencias ante la primera noticia, ya que en tanto se organiza la respuesta in situ, el centro sanitario ya está recibiendo víctimas.

Por añadidura es necesario conocer la realidad del entorno de los centros sanitarios, para en función del análisis de riesgos establecer un mapa de riesgos que determine la amenaza potencial para el centro sanitario, y por tanto el nivel de preparación deseable del mismo frente a un incidente de estas características. Destacándose la importancia del centro coordinador como regulador de flujos hacia los centros sanitarios preparados para atender a este tipo de víctimas, así como la importancia de una adecuada gestión de stocks de productos farmacológicos específicos para poder atender a las víctimas de un incidente $\mathrm{NBQ}^{25,26}$.

\section{PREPARACIÓN HOSPITALES ANTE INCIDENTES NBQ}

En función del agente causal que haya provocado el incidente NBQ, y fundamentalmente en ambiente químico, hay que tener en cuenta que pueda haber un peligro de transferencia de contaminación al conjunto de la cadena de rescate, incluido la contaminación secundaria del personal que atiende a las víctimas en el centro sanitario, priorizando en ambiente químico la descontaminación sobre el tratamiento ${ }^{27}$, independientemente de la clasificación asignada ${ }^{28}$, clasificación que resulta más complicada si cabe por el estado mental que presentan las víctimas ${ }^{29}$. Lo cual significa que las víctimas deban descontaminarse antes de la entrada al centro sanitario, de ahí que el hospital deba disponer de este tipo de instalaciones, fijas o portátiles, denominadas de for- 
ma genérica unidades de descontaminación o áreas de descontaminación química, o por el contrario integrar dentro de su plan de emergencias unidades de descontaminación, sanitarias o no, de servicios externos para así reducir el nivel de contaminación hasta un nivel aceptable y no constituyan un peligro para ellos y para los que les rodean (Figura 1).

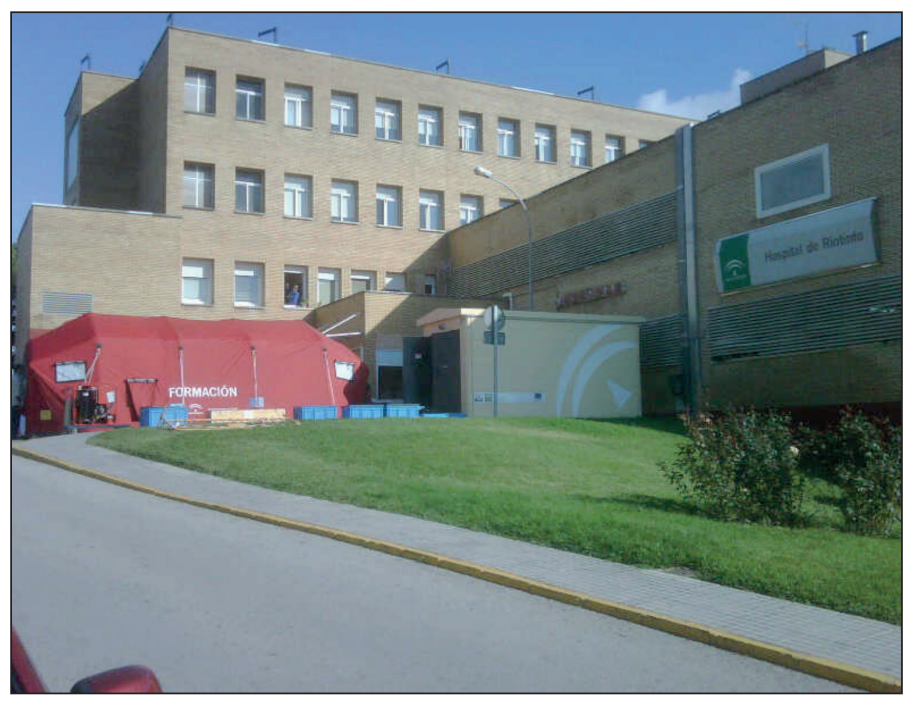

Figura 1. Estación de Descontaminación NBQ Hospitalaria.

Además, la transferencia de la contaminación motiva que en un incidente NBQ con múltiples víctimas el área de urgencias en vez de abrirse al interior, deba abrirse hacia el exterior gracias al establecimiento de unas áreas de expansión en función del estado clínico que presenten las víctimas para evitar la contaminación de la instalación.

La activación del plan de emergencias, junto con el establecimiento de las áreas de expansión deberá ser lo más rápida posible, debiendo tener en cuenta que a pesar de que se considera deseable que la activación de la unidad de descontaminación sea inferior a los 5 minutos, hay que considerar que esto en la práctica resulta muy complicado cuando se trate de unidades portátiles $^{30}$. Debiendo tener en cuenta que la activación deberá tener en cuenta los siguientes aspectos ${ }^{31}$ :

- Necesidad de realizar una activación y movilización activa de los recursos disponibles, tanto los humanos como los materiales necesarios en este tipo de incidentes.

- Disponer de un enlace con los Servicios de Emergencia Médicos o con la organización prehospitalaria que se trate.

- Agilizar la gestión de los enfermos hospitalizados y del flujo de víctimas.

- Gestión y atención sanitaria que requieran las víctimas.

- Activar y agilizar las evacuaciones secundarias.

- Establecer los flujos de información actualizada a las autoridades y familiares de las víctimas

En la figura 2 se puede ver la distribución conceptual de las áreas de expansión, mientras que en la figura 3 se puede observar un ejemplo imaginario de cómo podría organizarse ese área en un centro sanitario, observándose como la asistencia se lleva a vanguardia, estableciéndose una única ruta de entrada que lleva a la zona de Descarga de Ambulancias (DA) donde se continua con el proceso de tiraje continuo, así como se establece el Control de Contaminación (CC), en caso de que así se establezca, dotados con medios de detección o identificación, ya sea con medios propios o con medios de otros organismos (Cuerpos y Fuerzas de Seguridad, Bomberos, Servicios de Emergencia Sanitarios, etc.), para así, siempre que sea posible clasificar a las víctimas en función de si están contaminados o no. Este hecho no es baladí, ya que permite distinguir a los enfermos psicosomáticos (worried well), lo cual reducirá la carga de trabajo del personal destinado en las áreas de expansión y en la propia unidad de descontaminación (U. DECO). Motivo por el cual, los que no estén contaminados podrán pasar directamente a la zona de asistencia sanitaria, mejorando el pronóstico de las víctimas ${ }^{32}$.

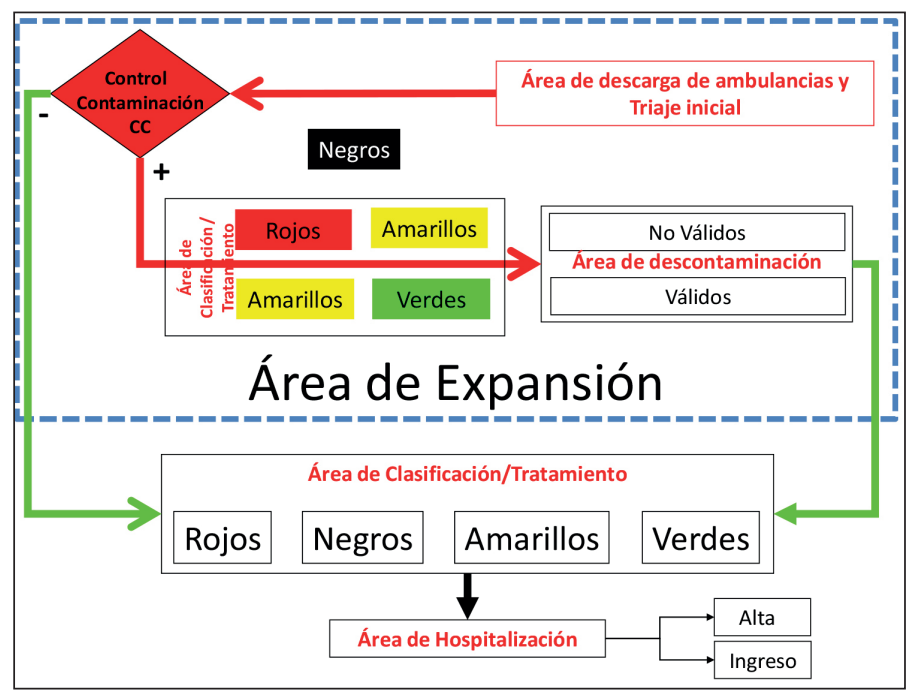

Figura 2. Esquema conceptual organización área de urgencias en incidentes $N B Q$.

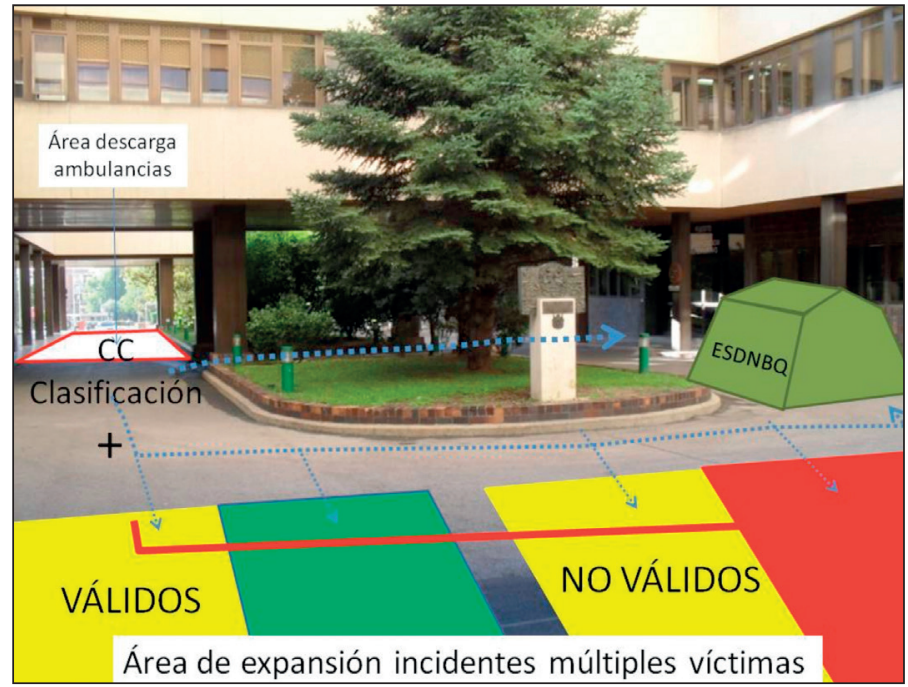

Figura 3. Ejemplo práctico organización área de expansión en incidentes $N B Q$.

Aquellas víctimas que hubieran resultado contaminadas serán ubicadas en las áreas de expansión en función de su grado de dependencia, así en el área de expansión de los no válidos se ubicarán los clasificados como rojos y amarillos, mientras que 
en la zona de válidos se ubicarán a los clasificados como amarillos y verdes, mientras que los negros se localizarán en una zona aparte vigilada. En estas zonas de expansión, se asistirá a las víctimas en función de las capacidades y de los procedimientos establecidos, teniendo en cuenta que estas áreas de expansión en realidad se tratan de válvulas de regulación que controlan el flujo de entrada hacia la unidad de descontaminación y por tanto hacia el área de urgencias.

Todos los vehículos que hayan transportado víctimas al centro sanitario deberán pasar obligatoriamente por la Estación de Descontaminación Vehicular (EDV) para ser controlada la contaminación, y ser descontaminados en caso necesario. Aunque hay que tener en cuenta que esto no es tarea ni obligación del centro sanitario, sino que es un claro ejemplo de la integración de la respuesta a incidentes NBQ.

Asimismo, el que exista riesgo de transferencia de contaminación significa que el personal de urgencias (así como todo el personal de intervención) debe adoptar el adecuado nivel de protección individual, en función del agente causal. Además, el personal sanitario debe estar entrenado para poder atender a víctimas que además de estar contaminadas, presentan traumatismos que complican su asistencia.

Y por otro lado, es necesario que disponga de los recursos sanitarios a un nivel dado en función del análisis de riesgos o de la dotación asignada, en caso de estar estas disponibles, así como de materiales y equipos especiales para tratar a este tipo de pacientes, ya sean respiradores multicanal, útiles para la descontaminación o salas dotadas con sistema de ventilación forzada sin recirculación de aire (siendo deseable que dispongan de filtros NBQ para que el aire que sale de la instalación esté libre de contaminantes), sistemas de duchas (y consiguiente recogida de efluentes), así como equipos de protección individual corporal y respiratoria frente a riesgo químico, biológico o radiológico $^{33-37}$.

Aunque todo esto no servirá para nada si el personal no dispone de la formación necesaria para poder intervenir en este tipo de incidentes, lo cual significa que deban realizarse actividades formativas específicas e integrar dentro de los procedimientos del centro sanitario actividades de entrenamiento mediante simulacros $\mathrm{y}$ ejercicios para alcanzar la capacidad necesaria y suficiente para ser capaz de dar un respuesta eficaz en este tipo de incidentes.

\section{EVALUACIÓN DE LA SEGURIDAD HOSPITALARIA ANTE INCIDENTES NBQ}

Al objeto de calificar, clasificar y adecuar los centros sanitarios en función del grado de seguridad (de continuar con su función) ante un posible desastre, la Organización Panamericana de la Salud ha implementado el llamado «Índice de Seguridad Hospitalaria» que es el valor numérico que expresa la probabilidad de que un establecimiento de salud existente continúe funcionando en casos de desastre, éste se refiere a la capacidad de resistencia de las instalaciones y al nivel de preparación de la organización ante emergencias masivas y desastres, así como la medida del grado de implementación del plan hospitalario para casos de desastres ${ }^{38,39}$.

Motivo por el cual parece razonable, aunque no haya sido específicamente diseñado para ello aplicarlo al análisis de la vulnerabilidad de la instalación (y sus ocupantes) ante un incidente NBQ. Este hecho ya era tenido en cuenta por la «Joint Comission on Accreditation of Healthcare Organizations» a partir del año 2000 al requerir que los hospitales tienen que tener un plan de manejo de pacientes contaminados, pero no las recomendaciones para proteger al personal a cargo de los pacientes contaminados ${ }^{40}$.

Para el cálculo del Índice de Seguridad Hospitalaria se dispone de una herramienta que determina dicho índice, habiéndose establecido tres categorías de instalaciones en función del nivel de seguridad existente. De esta manera, los centros sanitarios de categoría A funcionarán en situaciones de desastre sin poner en peligro a sus ocupantes, los centros de categoría B son aquellos que

Tabla 1. Capacidad funcional Centros Sanitarios ante incidentes NBQ.

\begin{tabular}{|c|c|c|c|c|c|}
\hline \multirow{2}{*}{ Plan operativo Incidentes NBQ } & \multicolumn{3}{|c|}{ Grado de seguridad } & \multirow{2}{*}{ OBS } & \multirow{2}{*}{ INSTRUCCIONES } \\
\hline & BAJO & MEDIO & ALTO & & \\
\hline Contempla crisis sociales y terrorismo & $\square$ & $\square$ & $\square$ & & \multirow{4}{*}{$\begin{array}{l}\text { B: Sin documento o sólo documento } \\
\text { M: Existe el plan y el personal } \\
\text { capacitado } \\
\text { A: Plan, personal capacitado y } \\
\text { recursos }\end{array}$} \\
\hline $\begin{array}{l}\text { Contempla emergencias químicas o } \\
\text { radiaciones ionizantes }\end{array}$ & & & & & \\
\hline Contempla agentes con potencial epidémico & & & & & \\
\hline \multicolumn{5}{|l|}{$\begin{array}{l}\text { Refuerzo de los servicios esenciales del } \\
\text { hospital }\end{array}$} & \\
\hline $\begin{array}{l}\text { Procedimiento para admisión de } \\
\text { emergencias y desastres }\end{array}$ & ᄃ & $\square$ & ᄃ & & \multirow{6}{*}{$\begin{array}{l}\text { B: No existe el procedimiento. } \\
\text { M: Existe el procedimiento y personal } \\
\text { entrenado } \\
\text { A: Procedimiento, personal } \\
\text { capacitado y recursos }\end{array}$} \\
\hline $\begin{array}{l}\text { Procedimiento de expansión área urgencias } \\
\text { y otras áreas críticas }\end{array}$ & $\square$ & $\square$ & L & & \\
\hline $\begin{array}{l}\text { Procedimientos para } \\
\text { epidemiológica intra-hospitalaria }\end{array}$ & & & & & \\
\hline $\begin{array}{l}\text { Procedimientos para la habilitación de sitios } \\
\text { para ubicación temporal de cadáveres y } \\
\text { medicina forense }\end{array}$ & & & & & \\
\hline \multicolumn{5}{|l|}{$\begin{array}{l}\text { Procedimientos para triaje, reanimación, } \\
\text { estabilización y tratamiento }\end{array}$} & \\
\hline \multicolumn{5}{|l|}{$\begin{array}{l}\text { Procedimientos de información al público y la } \\
\text { prensa }\end{array}$} & \\
\hline $\begin{array}{l}\text { Procedimientos para habilitación de espacios } \\
\text { para aumentar la capacidad, incluyendo la } \\
\text { disponibilidad de camas }\end{array}$ & & & & & $\begin{array}{l}\text { B: No identificadas áreas expansión. } \\
\text { M: Identificadas áreas y el personal } \\
\text { A: Identificadas áreas, personal y } \\
\text { recursos }\end{array}$ \\
\hline
\end{tabular}


tras un desastre han permanecido en pie, pero cuyo equipamiento y servicios estarán en riesgo. Mientras que los centros de categoría C tendrán una elevada probabilidad de que dejen de funcionar ya que no garantizan la vida y seguridad de los ocupantes ${ }^{41,42}$.

Saber el nivel de preparación y seguridad permite conocer las fortalezas y debilidades del centro para en el primer caso potenciarlas, y en el segundo caso establecer procedimientos de mejora continua al objeto de reducir el riesgo y vulnerabilidad de los establecimientos de salud. De ahí que desde el punto de vista de vulnerabilidad a un incidente NBQ haya que tener en cuenta alguno de los siguientes aspectos para así determinar el citado índice en función del grado de seguridad medido.

De hecho la inclusión, a pesar de los problemas de organización y gestión, del riesgo NBQ en el Plan de Emergencias o de Catástrofes externas es uno de los aspectos más importantes y más difíciles, desde el punto de vista de gestión, coordinación e integración, a la hora de establecer el índice de seguridad hospitalaria, así como el nivel de preparación para emergencias NBQ del personal que trabaja en el hospital y el grado de implementación del plan hospitalario para casos de desastre ${ }^{43}$. Para ello se tendrán en cuenta los aspectos relacionados en la tabla 1 en función de la capacidad funcional.

Otros aspectos muy importantes que hay que tener en cuenta a la hora de evaluar el índice es la integración del plan dentro de otros planes, ya sean a nivel municipal como autonómico o nacional, evaluándose la no vinculación con un grado de seguridad bajo, vinculado no operativo con un grado medio y vinculado y operativo con un grado de seguridad alto.

La realización de ejercicios de simulación o simulacros es uno de las variables a evaluar más importantes dentro del sistema de calidad, ya que como dice el aforismo de Benjamin Franklin «Dime y lo olvido, enséñame y lo recuerdo, involúcrame y lo aprendo», con lo cual se consigue que el personal se implique en el plan de mejora continua y se mejore en la respuesta a emergencias. De esta manera si no se realizan ejercicios se evaluará con un índice bajo, mientras que si hay planes y el personal está capacitado el índice será medio, y si por añadidura hay recursos disponibles para llevar a cabo ejercicios y simulacros se calificará con un índice alto.

\section{CONCLUSIÓN}

La experiencia demuestra que los centros sanitarios sufren las consecuencias de un incidente NBQ, ya sea natural o provocado, de forma directa, incluso con una única víctima, planteándose la necesidad de establecer centros de referencia especializados donde se dirijan los flujos de evacuación regulados, aunque esto no es óbice para potenciar la preparación de los centros hospitalarios para hacer frente a este tipo de incidentes. Debiendo el centro realizar un esfuerzo colectivo e individual para poder hacer frente a un incidente no convencional, tanto con medios organizativos como materiales mediante la inclusión dentro del plan de catástrofes del riesgo NBQ y la realización de ejercicio y simulacros que mejoren el nivel de preparación de la instalación. El problema es que ese esfuerzo colectivo, e individual, en la actualidad está fuera del alcance de la mayoría de los centros sanitarios.
A pesar de que todo el mundo habla de la coordinación e integración de los centros sanitarios en los Planes de Catástrofes externas resulta muy complicado pasar de la teoría a la práctica. Este hecho no es baladí, ya que esa potencial integración es un factor determinante a la hora de establecer, en el caso de que fuera necesario, el índice de seguridad hospitalario. Motivo por el cual se demuestra como vital el papel del centro coordinador como regulador de flujos en un incidente NBQ, toda vez que se siguen produciendo el mismo número de patologías que en un día cualquiera.

La respuesta a un incidente NBQ debe ser multidisciplinar, gradual y progresiva, donde toda la cadena sanitaria pueda responder con eficacia a un incidente de estas características, iniciándose con la existencia de un centro coordinador eficaz, unos servicios de emergencia extrahospitalarios formados y entrenados, así como integrados con los servicios de emergencia no sanitarios que puedan controlar los flujos de víctimas hacia centros sanitarios de referencia especializados o no en la atención de los afectados en un incidente no convencional como puede ser un incidente NBQ.

\section{BIBLIOGRAFÍA}

1. International Atomic Energy Agency - IAEA. The Radiological Accident in Goiânia STI/PUB/815. IAEA, Vienna, 1988.

2. Dirección General de Medios de Comunicación de la Comunidad de Madrid. El consejero Güemes visita este centro, único en España, en su XXV Aniversario. Comunicado de prensa. Comunidad de Madrid, 30/09/08 (consultado 27/04/12). Disponible en: http://www.madrid.org/cs/Satellite?blobcol=urldat a\&blobheader $=$ application $\% 2$ Fpdf \&blobheadername $1=$ Content-dispositio n\&blobheadername $2=$ cadena\&blobheadervalue $1=$ filename $\% 3 \mathrm{DNP}+$ Visita + consejero + al + centro + de + radiopatolog $\% \mathrm{C} 3 \%$ ADa.pdf\&blobheadervalue 2 $=$ language $\% 3 \mathrm{Des} \% 26$ site $\% 3 \mathrm{DH}$ ospitalGregorioMaranon\&blobkey= id \&bl obtable $=$ MungoBlobs\&blobwhere $=1220365031953 \&$ ssbinary $=$ true

3. Bayo I. Entrevista a Rafael Herranz Crespo, director del Centro de Radiopatología y Radioprotección del Hospital Gregorio Marañón. Alfa Revista de seguridad nuclear y protección radiológica del Consejo de Seguridad Nuclear. III trimestre 2011;15:24-29.

4. Portal de Salud de la Comunidad de Madrid. El Marañón analiza los aspectos sanitarios y organizativos en emergencias nucleares 01/02/2012 (consultado 27/04/12). Disponible en: http://www.madrid112.es/cs/Satelli te cid $=1142673252044 \&$ language $=$ es $\&$ pageid $=1159444389315 \&$ pagename $=$ PortalSalud\%2FCM_Actualidad_FA\%2FPTSA_pintarActualidad\&pestann $\mathrm{a}=3 \&$ vest $=1159444389315$

5. Nakajima T, Sato S, Morita H, Yanagisawa N. Sarin poisoning of a rescue team in the Matsumoto sarin incident in Japan. Occupational and Environmental Medicine 1997;54:697-701.

6. Nozaki H, Hori S, Shinozawa Y, Fujishima S, Takuma K, Sagoh M, Kimura H, Ohki T, Suzuki M, Aikawa N. Secondary exposure of medical staff to sarin vapor in the emergency room. Intensive Care Med. 1995 Dec;21(12):1032-5.

7. Okumura S, Okumura T, Ishimatsu S, Miura K, Maekawa H, Naito T. Clinical review: Tokyo - protecting the health care worker during a chemical mass casualty event: an important issue of continuing relevance. Cri Care 2005;9(4):397-400.

8. U.S National Research Council. Acute Exposure Guideline Levels for Selected Airborne Chemicals, Volume 3. U.S National Research Council. Subcommittee on Acute Exposure Guideline Levels, Committee on Toxicology. Board on Environmental Studies and Toxicology - National Research Council. The National Academies Press. 2003:44-49.

9. Masci JR, Bass E. Bioterrorism: A guide for Hospital Preparedness. CRC Press 2005:29-46

10. Rodríguez Soler AJ, León González JS, Garijo Gonzalo G, Peláez Corres N, Castro Delgado R, et al. El sistema de triage ante brotes pandémicos emer- 


\section{Preparación Hospitalaria en incidentes NBQ}

gentes Tri@ge Revista electrónica. 2010;1:1 (consultado 28/04/12). Disponible en: http://www.cetph.es/CETPH/revista1.html

11. Cique Moya A, Serrano López MC, Rodríguez Soler AJ. Gripe y Equipos de Protección Individual. Prehospital Emergency Care (ed. esp.).2009; 02(03):231-7.

12. Croft J, Bailey M, Maguire H, Tattersall P, Morrey M, et al. Management of Response to the Polonium-210 Incident in London. Proceedings of 12th Congress of the International Radiation Protection Association. Buenos Aires, October $19-24,2008$ (consultado 28/04/12). Disponible en: http://www. irpa12.org.ar/fullpapers/FP0841.pdf

13. Mercadal G, Pastó L, Palom FJ, Parayre M. Utilización de pralidoxima en la intoxicación por organofosforados y carbamatos. Revista de la OFIL. 2004,14;4:53-61

14. Perayre M, Leiva E, Pasó L, Jódar R. Síndrome intermedio en el transcurso de una intoxicación por organofosforados a pesar de una infusión continua de pralidoxima. An. Med. Interna (Madrid) 2007;24(3)129-131.

15. Barco JC, Omar C, Puiguriguer J, Coll I, et al. Revisión de las intoxicaciones graves por insecticidas organofosforados en un período de 11 años (19962006). Emergencias 2008;20:207-211.

16. White SM. Chemical and biological weapons. Implications for anaesthesia and intensive care. Br. J. Anaesth. 2002;89(2):306-324.

17. Danzig R, Sageman M, Leighton T, Hough Ll, et al. Aum Shinrikyo Insights Into How Terrorists Develop Biological and Chemical Weapons Center for a New American Security. July 2011.

18. U.S National Research Council. Acute Exposure Guideline Levels for Selected Airborne Chemicals, Volume 3. U.S National Research Council. Subcommittee on Acute Exposure Guideline Levels, Committee on Toxicology. Board on Environmental Studies and Toxicology - National Research Council. The National Academies Press. 2003:44-49.

19. Alvarez Leiva C. Manual de Atención a Múltiples Víctimas y Catástrofes. Arán Ediciones, 2005:317-339.

20. Ministerio de Sanidad y Política Social. Manual de procedimientos y recomendaciones para la atención sanitaria urgente en incidente químicos accidentales o terroristas (Coordinador de la Edición, Antonio Dueñas Laita). Ministerio de Sanidad y Política Social 2009:57:66.

21. MMWR Nosocomial Poisoning Associated With Emergency Department Treatment of Organophosphate Toxicity --- Georgia, 2000 January 05, 2001/49(51);1156-8 MMWR weekly, January 05, 2001. (consultado 01/05/12). Disponible en: http://www.cdc.gov/mmwr/preview/mmwrht$\mathrm{ml} / \mathrm{mm} 4951 \mathrm{a} 2 . \mathrm{htm}$

22. Aschkenasy-Steuer G, Shamir M, Rivkind A, Mosheiff R, Shushan Y, et al. Clinical Review: The Israeli experience: conventional terrorism and critical care. Critical Care 2005; 9: 490-499.

23. Gutiérrez de Ceballos JP, Turégano F, Pérez Díaz D, Sanz Sánchez M, Martín Llorente C, Guerrero Sanz JE. Casualties treated at the closest hospitals in the Madrid,March 11, terrorist bombing. Critical Care Medicine 2005; 33(1S): s107-s112.

24. Pita Pita R. La gestión de crisis químicas. En: Realidades y Perspectivas de la Gestión Internacional de Crisis. Adenda 2008. Compilador Enrique Vega Fernández. Instituto Universitario «General Gutiérrez Mellado» de Investigación sobre la Paz, la Seguridad y la Defensa. 2008:201-222.

25. González M. España amplía al carbunco su reserva de vacunas contra el bioterrorismo. Diario El País 8/02/2003 (consultado 26/06/2012). Disponible en url: http://elpais.com/diario/2003/02/08/espana/1044658810_850215.html

26. Pita R, Ishimatsu S, Robles R. Actuación sanitaria en atentados terroristas con agentes químicos de guerra: más de diez años después de los atentados con sarín en Japón ( $2^{\mathrm{a}}$ parte). Emergencias 2007;19:337-346
27. Cique Moya A. Emergencias NBQ. Pautas de Intervención Sanitaria. Editorial MARBAN 2009:86-92.

28. Nogué S, Amigó M, Uría E, Fernández F, Velasco V. Actividad de un área de descontaminación química en un servicio de urgencias. Emergencias 2012:24:203-207.

29. Nogué S, Ramos A, Portillo M, Bohils M. Adecuación de un sistema de triaje y del circuito asistencial en urgencias al paciente intoxicado. Emergencias 2010;22:338-344.

30. Macintyre AG, Christopher GW, Eitzen E, Gum R, Weir S, DeAtley C, Tonat K, Barbera JA. Weapons of Mass Destruction Events With Contaminated Casualties Effective Planning for Health Care Facilities. JAMA. 2000;283:242-249.

31. Organización Panamericana de la Salud. Establecimiento de un sistema de atención a víctimas en masa. 1996:35

32. Shapira Y, Bar Y, Berkenstadt, Atsmon J, Danon YL. Outline of Hospital Organization for a Chemical Warfare Attack. En:Chemical Warfare Medicine Aspects and Perspectives from the Persian Gulf War. Editors Y.L. Danon, J. ShemerGefen Publishing House Limited 1994:144-151.

33. Organización Panamericana de la Salud- OPS. Accidentes Químicos: Aspectos relativos a la salud. Guía para la preparación y respuesta.OPS, Programa Internacional de Seguridad sobre sustancias químicas, Organización de Cooperación y Desarrollo Económico, Programa de las Naciones Unidas para el Medio Ambiente. Centro de Actividades de Programas para la Industria y el Medio Ambiente, Organización Mundial de la Salud - Centro Europeo para el Medio Ambiente y la Salud.1998

34. Collado Coello AK, González Gámiz G, Gómez Carril M. Los antídotos en la lucha contra las intoxicaciones. Rev Cubana Farm 2004;38(2). (Consultado 02/05/12). Disponible en: http://bvs.sld.cu/revistas/far/vol38_2_04/ far10204.htm

35. Aguilar Salmerón R, Soy Muner D, Nogué Xarau S. Disponibilidad de antídotos en los ámbitos sanitarios de Cataluña. Med Clin (Barc). 2006;127(20):770-3.

36. Cáceres P. Ropa de Protección: Requisitos generales. Instituto Nacional de Seguridad e Higiene en el Trabajo. Notas Técnicas de Prevención 769

37. Prieto JM, Hernández A. Equipos de protección respiratoria: identificación de los filtros según sus tipos y clases. Instituto Nacional de Seguridad e Higiene en el Trabajo. Notas Técnicas de Prevención 787.

38. Hospitales Seguros Frente a los Desastres. Campaña Mundial 2008-2009 para la Reducción de Desastres. Estrategia Internacional para la Reducción de Desastres, Organización Mundial de la Salud, El Banco Mundial. 2008 (consultado 01/05/12). Disponible en: http://www.unisdr.org/2009/campaign/ pdf/wdrc-2008-2009-information-kit-spanish.pdf

39. Organización Panamericana de la Salud. Hospitales Seguros. Organización Panamericana de la Salud. (consultado 01/05/12). Disponible en: http://www.paho.org/spanish/dd/ped/HospitalesSeguros.pdf

40. Geller RJ, Singleton KL, Tarantino ML, Drenzek CL, Toomey KE. Nosocomial Poisoning Associated With Emergency Department Treatment of Organophosphate Toxicity—Georgia, 2000. MMWR. 2001;49:1156-1158. En: JAMA, 2001;285(5):527.

41. http://www.paho.org/spanish/dd/ped/SafeHospitalsChecklist.htm

42. Organización Panamericana de la Salud. Índice de Seguridad Hospitalaria: Formularios para la evaluación de hospitales seguros. Organización Panamericana de la Salud.2008.

43. Alted López E, Cuenca Solana M, Durá Jimenez MJ, Hernando Lorenzo JE, Kessler Saiz P et al. Plan de Catástrofes Externas del Hospital Universitario 12 de octubre. Hospital Universitario 12 de octubre, junio 2007. 